\title{
Rapid clinical evolution for COVID-19 translates into early hospital admission and unfavourable outcome: a preliminary report
}

\author{
Markus Heim, ${ }^{1}$ Tobias Lahmer, ${ }^{2}$ Sebastian Rasch, ${ }^{2}$ Silja Kriescher, ${ }^{1}$ Wiebke Berg-Johnson, ${ }^{1}$ Kristina Fuest, ${ }^{1}$ Barbara \\ Kapfer, ${ }^{1}$ Gerhard Schneider, ${ }^{1}$ Christoph D. Spinner, ${ }^{2,3}$ Fabian Geisler, ${ }^{2}$ Johannes R. Wießner, ${ }^{2}$ \\ Kathrin Rothe, ${ }^{4}$ Susanne Feihl, ${ }^{4}$ Andreas Ranft ${ }^{1}$ \\ ${ }^{1}$ Department of Anaesthesiology and Intensive Care Medicine, University hospital rechts der Isar, Technical University of Munich, \\ School of Medicine, Munich \\ ${ }^{2}$ Department of Internal Medicine II, University hospital rechts der Isar, Technical University of Munich, School of Medicine, Munich \\ ${ }^{3}$ German Centre for Infection Research (DZIF), Partner Site Munich, Munich \\ ${ }^{4}$ Institute for Medical Microbiology, Immunology and Hygiene, Technical University of Munich, School of Medicine, Munich, \\ Germany
}

Background: A wide range of mortality rates has been reported in COVID-19 patients on the intensive care unit. We wanted to describe the clinical course and determine the mortality rate in our institution's intensive care units.

Methods: To this end, we performed a retrospective cohort study of 50 COVID-19 patients admitted to the ICU at a large German tertiary university hospital. Clinical features are reported with a focus on ICU interventions, such as mechanical ventilation, prone positioning and extracorporeal organ support. Outcome is presented using a 7-point ordinal scale on day 28 and 60 following ICU admission.

Results: The median age was 64 years, $78 \%$ were male. LDH and D-Dimers were elevated, and patients were low on Vitamin D. ARDS incidence was $75 \%$, and 43/50 patients needed invasive ventilation. 22/50 patients intermittently needed prone positioning, and 7/50 required ECMO. The interval from onset of the first symptoms to admission to the hospital and to the ICU was shorter in non-survivors than in survivors. By day 60 after ICU admission, 52\% of the patients had been discharged. 60-day mortality rate was 32\%; $37 \%$ for ventilated patients, and $42 \%$ for those requiring both: ventilation and renal replacement therapy.

Conclusions: Early deterioration might be seen as a warning signal for unfavourable outcome. Lung-protective ventilation including prone positioning remain the mainstay of the treatment.

Key words: COVID-19; SARS-CoV-2; critical care; mortality; acute respiratory distress syndrome; prone position; invasive ventilation; retrospective cohort study.

Correspondence: Dr. med. Markus Heim, Technical University of Munich, School of Medicine, University Hospital rechts der Isar, Department of Anaesthesiology and Intensive Care Medicine, Ismaninger Str. 22, 81675 Munich, Germany.

Tel. +49.89.41405990 - Fax: +49.89.41404804. E-mail: m.heim@tum.de

Contributions: $\mathrm{MH}, \mathrm{AR}$, conception and design, analysis and interpretation of data, drafting the article, final approval of the version to be published, agreement to be accountable for all aspects of the work; TL, SR, SK, WBJ, KF, BK, GS, CDS, FG, JRW, KR, SF, analysis and interpretation of data, revising the article for important intellectual content, final approval of the version to be published, agreement to be accountable for all aspects of the work.

Conflict of interest: Susanne Feihl reports personal fees and non-financial support from Smith and Nephew, personal fees and nonfinancial support from Curetis, personal fees and non-financial support from Zimmer Biomet, personal fees and non-financial support from Limbach, personal fees and non-financial support from MSD, outside the submitted work; Sebastian Rasch reports travel-grants from Gilead, outside the submitted work. Christoph D. Spinner reports grants from Aperion, grants and personal fees from Gilead Sciences, grants and personal fees from MSD, grants and personal fees from GSK/ViiV Healthcare, grants and personal fees from Janssen-Cilag, personal fees from Molecular Partners, personal fees from Formycon, from null, outside the submitted work. All other authors (Wiebke Berg-Johnson; Kristina Fuest; Fabian Geisler; Markus Heim; Barbara Kapfer; Silja Kriescher; Tobias Lahmer; Kathrin Rothe; Gerhard Schneider; Johannes R. Wießner) have no conflict of interest to disclose.

Funding: This research did not receive any specific grant from funding agencies in the public, commercial, or not-for-profit sectors. Availability of data and materials: The complete anonymised set of individual patient data is available from the authors upon request.

Ethics approval and consent to participate: The Ethics Committee of the Technical University of Munich approved this retrospective study (approval no. 723/20 S-SR). 


\section{Introduction}

The coronavirus disease 2019 (COVID-19) outbreak, with its beginning in Wuhan in December 2019, led into a pandemic. While most patients develop mild or uncomplicated illness, other require hospitalisation, and of these about $15 \%$ need treatment on an intensive care unit (ICU) [1]. Complications such as acute respiratory distress syndrome (ARDS), sepsis, and multiorgan failure can occur [1-3]. With continuously growing knowledge about the pathogen, its transmission and the manifestations of this new disease, widely varying mortality rates have been reported. Reported clinical data are heterogeneous with respect to the number of patients treated and also the censoring day to determine outcome. For instance, in a cohort of 52 patients on a single ICU in Wuhan, the 28 -day mortality rate was $62 \%$, and up to $74 \%$ if ARDS was present [4]. Early reports from smaller cohorts in Seattle (WA, USA) showed ICU mortality rates between $50 \%$ ( 12 of 24 patients) and $67 \%$ (14 of 21 patients) rising up to $75 \%$ in patients on invasive ventilation $[5,6]$. These mortality rates for severe acute respiratory syndrome coronavirus 2 (SARS-CoV-2) induced ARDS are significantly higher than those in the 'LUNG SAFE' study from the pre COVID-19 era, which presented hospital mortality rates between $35 \%$ and $46 \%$ depending on the severity of ARDS [7]. In contrast, an in-hospital mortality of $36 \%$ (59 of 165 patients) was reported from Atlanta (GA, USA) [8]. It is widely acknowledged that mortality in critically ill patients is associated with the severity of illness on arrival to the ICU and the need for interventions such as mechanical ventilation, renal replacement therapy and vasopressor support $[9,10]$. Likewise, older age and the presence of comorbidities worsen outcome $[4,11,12]$. In COVID-19 patients, the presence of chronic lung diseases like asthma or chronic obstructive pulmonary disease (COPD), as well as obesity and persisting elevation of infection parameters are associated with an increased likelihood to develop ARDS [3].

The aim of our study was to characterise the COVID-19 ICU patients treated in our university hospital, to describe the interventions and the outcomes, and to identify differences between patients that had survived and those who had died until day 60 after ICU admission.

\section{Methods}

We performed a retrospective single-centre cohort study of 50 adult patients with confirmed COVID-19 during the first pandemic wave, admitted to the ICU between 11 March and 24 April 2020. Patients were treated on two different intensive care units in a university hospital with 1,163 beds, one affiliated to the department of internal medicine II and one to the department of anaesthesiology and intensive care medicine.

The Ethics Committee of the Technical University of Munich approved this retrospective study (approval no. 723/20 S-SR).

Medical records including clinical charts and nursing records were reviewed. Data collection included patient biometrics, comorbidities, clinical parameters, laboratory findings, information on inpatient management, ICU interventions, as well as ICU and hospital stay. On day 28 and day 60 from ICU admission, outcome was measured with a 7-point ordinal scale from category 1 (not hospitalized with no limitations on activities) to category 7 (dead) as used before [13].

Laboratory confirmation of SARS-CoV-2 was achieved by polymerase chain reaction (PCR) of respiratory swabs or combination of IgG/IgM-seropositivity and COVID-19 symptoms. Details to the PCR and serological testing methods are published elsewhere [14]. Additionally, a chest computed tomography (CT) scan was performed for nearly all patients (48/50) to identify typical CT findings for COVID-19 [15,16]. Patients with positive PCR or positive IgM/IgG serology results were defined as definite COVID-19 cases. Patients with suspected disease in whom diagnosis could not be confirmed were excluded from the analysis.

Continuous data are described by median (interquartile range from quartile $25 \%$ to quartile $75 \%$ ), and categorical data by absolute and relative frequencies. Data were analysed using a chisquare or Wilcoxon rank-sum test for categorical and continuous variables, respectively, with a two-sided p-value of less than 0.05 considered statistically significant. Due to the exploratory nature of the study, uncorrected p-values are reported. Statistical analyses were performed using Microsoft Excel 2013 and IBM SPSS Statistics ver. 25.0 (IBM Corp, Armonk, NY). The complete anonymised set of individual patient data is available from the authors upon request.

\section{Medical records}

In March and April 2020, 50 adult patients with a confirmed diagnosis of COVID-19 were admitted to ICU in our university hospital. Comprehensive datasets containing clinical and laboratory parameters were compiled from these cases. Thirty-three patients $(66 \%)$ had initially been treated on the normal ward before transfer to the ICU, 17 patients (34\%) were assigned directly to the ICU via the emergency department. At the time of symptom onset, 6 patients $(12 \%)$ were in hospital for a diagnosis other than COVID-19. Oxygen therapy was started when $\mathrm{SpO}_{2}$ dropped below 94\%. All COVID-19 patients received respiratory therapy by a physiotherapist at least once daily. According to our standard operating procedure for COVID-19 patients, the need for intensive care was discussed when the respiratory rate rose above 30 per min and $\mathrm{SpO}_{2}$ fell below $90 \%$ at an oxygen flow rate of $8 \mathrm{~L} / \mathrm{min}$ by face mask. An overview of the variables not presented in Tables 1 and 2 can be found the Tables S1 to S4 in the Supplementary Material. Presence of SARS CoV-2 was proven by PCR in 47 cases. In 42 out of 44 cases, serologic testing (IgG and IgM) indicated SARSCoV-2 infection, including all three cases with negative PCR. Imaging findings were consistent with COVID-19 in all patients receiving a chest-CT $\operatorname{scan}(\mathrm{n}=48)$.

\section{Patient characteristics}

The median age in the whole cohort was 64 (range, 26-96) years. Seventy-eight percent $(39 / 50)$ of patients were male. In ten patients (20\%), no prior comorbidity was documented. At least one underlying comorbidity was present in $38 / 50$ patients (76\%), with arterial hypertension being the most frequent (in 56\%). Three or more comorbidities were present in $36 \%$ of all patients (supplementary Table S1). The distribution of blood groups in the ABO system was similar between survivors and non-survivors, with comparable proportions of group $\mathrm{O}$.

After a median of four days (1-7) after onset of symptoms, patients were admitted to hospital, and one day (0-3) later, transferred to ICU. On admission to the ICU, sepsis-related organ failure assessment score (SOFA) score was six (3-10) and acute physiology and chronic health evaluation score (APACHE) was 18 (14$26)$. The majority of patients $(92 \%)$ was lymphopenic, and all showed elevated LDH and D-dimers. Median concentration of vitamin $\mathrm{D}\left(25-\mathrm{OH}\right.$-vitamin $\left.\mathrm{D}_{3}\right)$ was $14 \mathrm{ng} / \mathrm{mL}$; more than three quarters of our patients were low on vitamin $\mathrm{D}(£ 30 \mathrm{ng} / \mathrm{mL})$, and $42 \%$ presented a manifest deficiency (threshold, $12 \mathrm{ng} / \mathrm{mL}$ ). Hypalbuminaemia ( $£ 3.5 \mathrm{~g} / \mathrm{dL}$ ) was found in $70 \%$, with a median of $3.0 \mathrm{~g} / \mathrm{dL}$. Leukocytes, PCT and interleukin-6 did not show a 
Table 1. Demographic characteristics and laboratory findings on ICU admission.

\begin{tabular}{|c|c|c|c|c|}
\hline Characteristics, median (IQR) & All $(n=50)$ & Survivors* $(\mathrm{n}=34)$ & Non-survivors* $(n=16)$ & $\mathbf{p}^{\circ}$ \\
\hline Age 64 (53-77) & $59.5(53-75)$ & $71(54-81)$ & 0.174 & \\
\hline Male sex, n (\%) & $39(78)$ & $26(76)$ & $13(81)$ & 1.0 \\
\hline SOFA & $6(3-10)$ & $6(3-10)$ & $7(4.5-10.5)$ & 0.5552 \\
\hline Apache II & $18(14-25.5)$ & $17(14-23)$ & $21(14.5-34.5)$ & 0.215 \\
\hline \multicolumn{5}{|l|}{ Comorbidities } \\
\hline None, n (\%) & $10(20)$ & $8(23.5)$ & $2(12.5)$ & 0.4684 \\
\hline Any, n (\%) & $38(76)$ & $25(73.5)$ & $13(81.3)$ & 0.7278 \\
\hline$\geq 3, \mathrm{n}(\%)$ & $18(36)$ & $13(38.2)$ & $5(31.3)$ & 0.7568 \\
\hline \multicolumn{5}{|c|}{ Laboratory findings on ICU admission } \\
\hline Leucocytes (G/L) & $7.93(5.9-10.9)$ & $7.92(6.02-10.77)$ & $8.79(4.97-11.61)$ & 0.6527 \\
\hline Lymphocytes (\% of Leucs) & $9(5-13)$ & $9(7-12)$ & $8(4-14.5)$ & 0.984 \\
\hline $\mathrm{CRP}(\mathrm{mg} / \mathrm{dL})$ & $13.65(8.6-20.6)$ & $15.1(10.4-21)$ & $10.3(7.3-17.8)$ & 0.7114 \\
\hline Il6 (pg/mL) & $135(89-195)$ & $135(85-193)$ & $133(118-193)$ & 0.3125 \\
\hline PCT (ng/mL) & $0.3(0.1-0.9)$ & $0.3(0.2-1.4)$ & $0.3(0.1-0.8)$ & 0.5093 \\
\hline D-Dimer ( $\mu \mathrm{g} / \mathrm{L}$ FEU) & $1983(982-6614)$ & $2023.5(983-6578)$ & $1983(1093-6640)$ & 0.9442 \\
\hline $\mathrm{LDH}(\mathrm{U} / \mathrm{L})$ & $468(340-592)$ & $468(355-573)$ & $471(319-713.75)$ & 0.8026 \\
\hline Albumin $(\mathrm{g} / \mathrm{dL})$ & $3(2.6-3.5)$ & $3(2.7-3.5)$ & $3.1(2.6-3.8)$ & 0.5029 \\
\hline 25-OH-Vitamin $\mathrm{D}_{3}(\mathrm{ng} / \mathrm{mL})$ & $14(8.5-26.5)$ & $14(8-27)$ & $13(10.25-19.5)$ & 0.7642 \\
\hline
\end{tabular}

*Until outcome day 60 from ICU admission; ${ }^{\circ}$ Chi Square or Wilcoxon rank-sum test comparing those who survived vs died up to day 60 from ICU admission.

notable elevation. For four selected timepoints during the ICU stay (on day of admission, intubation, first proning and extracorporeal membrane oxygenation (ECMO) initiation), no significant changes in the laboratory parameters (leukocytes, CRP, I16, PCT, LDH and D-dimer) could be detected. Median time to first negative PCR was 16 days (10-22). Patient characteristics and initial laboratory data are shown in Table 1 and supplementary Table S1, course of laboratory data are shown in Supplementary Table S2.

\section{Treatment and course of disease}

Non-invasive ventilation (NIV) or high flow nasal oxygen therapy (high flow nasal oxygen, HFNO) was used in four cases but could not obviate the need for subsequent endotracheal intubation. $43(86 \%)$ of the patients received invasive ventilation. In more than $90 \%$, the Berlin criteria for diagnosis of ARDS were met on day of intubation, with $65 \%(\mathrm{n}=28)$ categorized as moderate or severe with a median Horovitz index (HI) of $160 \mathrm{mmHg}(113-216)$ [17]. On the day after intubation, there was no relevant change in severity of ARDS. After intubation, the median peak airway pressure was 25 mbar, with a median positive endexpiratory pressure (PEEP) of 10 mbar which was largely maintained on this level until the next day. Half of the ventilated patients $(n=22)$ needed prone positioning at a median HI of $87 \mathrm{mmHg}(72-107)$. During the first proning period (approximately 16 hours) the achieved average HI was $135 \mathrm{mmHg}(115-155)$, a clinically relevant and statistically significant increase ( $\mathrm{p} £ 0.01)$ (Figure 1). After a median of nine days (6-11) on invasive ventilation, seven patients underwent veno-venous ECMO. On day of ECMO initiation, the median SOFA score was 10 (10-11).

During hospital stay, nine patients of our ICU cohort received remdesivir within clinical studies or early access programs, and six patients received convalescent plasma. Dexamethasone (or any other glucocorticoid) were not administered routinely, as no general recommendation was available at the time of the study period. In our hospital, lopinavir/ritonavir, chloroquine or hydroxychloro-
Table 2. Outcome on day 28 and 60 after ICU admission.

\begin{tabular}{llcc}
$\begin{array}{l}\text { Scale } \\
\text { value }\end{array}$ & Description & $\begin{array}{c}\text { Day 28 } \\
\text { n (\%) }\end{array}$ & $\begin{array}{c}\text { Day 60 } \\
\text { n (\%) }\end{array}$ \\
1 & Not hospitalized, no limitation on activities & $6(12)$ & $10(20)$ \\
2 & Not hospitalized, limitation on activities & $11(22)$ & $16(32)$ \\
\hline 3 & Hospitalized, not requiring supplemental oxygen & $3(6)$ & $1(2)$ \\
4 & Hospitalized, requiring supplemental oxygen & $3(6)$ & $3(6)$ \\
\hline 5 & Hospitalized, on non-invasive ventilation or high flow & $2(4)$ & $0(0)$ \\
6 & Hospitalized, on invasive ventilation or ECM0 & $14(28)$ & $4(8)$ \\
\hline 7 & Dead & $11(22)$ & $16(32)$ \\
\hline
\end{tabular}

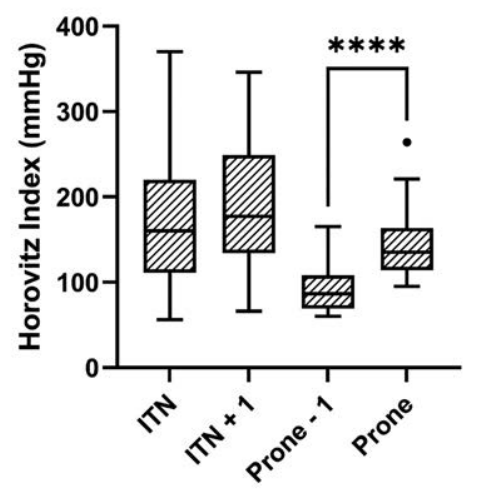

Figure 1. Oxygenation (expressed as Horovitz Index) on day of intubation ('ITN', $n=43$ ), one day later ('ITN +1 ', $n=39$ ), before proning (prone $-1, n=20$ ), and in prone position (prone, $n=20$, average of repeated measurements). In prone position, oxygenation improved significantly (prone -1 vs prone, $\mathrm{p}<0.0001$, Wilcoxon matched-pairs signed rank test). Box and whiskers are median, lower/upper quartile, and 1.5 times interquartile range. 
quine were used for potential treatment of COVID-19. 84\% of all patients needed vasopressor support. 20 patients (40\%) underwent renal replacement therapy (Supplementary Table S4). As described recently, severe bacterial and fungal co-infections were rare in our cohort [14]. During their hospital stay, 48 patients (96\%) were treated with antibiotics. Bloodstream infections on the day of admission were rare and could be detected in a total of 14 patients (28\%) during the course of intensive care therapy. For prevention of thromboembolism, all patients received low-molecular-weight heparins in double prophylactic dosage, with the exception of patients for whom therapeutic anticoagulation was indicated (e.g., due to ECMO therapy).

\section{Results}

Median length of stay on the ICU was 17 days (9-38). Ventilated patients spent 19 days (11-42) on the ICU with an average time on the ventilator of 18 days (6-11). Among the 31 patients that could be discharged from the ICU, 22 were transferred to a regular ward, 4 were transferred to another ICU or a weaning facility, 4 to rehabilitation centre, and 1 patient could return home. On day $60,52 \%$ of our patients $(\mathrm{n}=26)$ had been discharged home, and $38 \%$ of them ( $\mathrm{n}=10$, a fifth of the whole cohort) reported no limitations on activities. Four ( $8 \%$ ) patients were still on ICU on day 60 , one of them still being on ECMO. Sixty-day mortality rate was
$32 \%$ for all patients, $37 \%$ for the patients on ventilator and $42 \%$ for patients requiring both mechanical ventilation and renal replacement therapy. Four of seven patients treated with ECMO died, three of them due to fatal intracerebral haemorrhage. One patient was still on ECMO on day 60 (Table 2).

Factors associated with adverse outcome in COVID-19 have repeatedly been described, among them, age, obesity and comorbidities $[18,19]$. For further exploratory analysis, we divided our cohort into two subgroups: Patients who survived $(n=34)$ or died $(n=16)$ up to day 60 after ICU admission. Between these groups, age, body mass index and number of comorbidities did not differ significantly. A large proportion of survivors (76\%) and all $(100 \%)$ non-survivors needed vasopressor support $(\mathrm{p}=0.04)$. Patients not surviving to day 60 after ICU admission received dialysis more frequently (50\% vs 35\%), although this was not statistically significant. Counted from the first onset of symptoms, non-survivors were admitted earlier than survivors - both to hospital and to ICU (Figure 2 A,B and Supplementary Table S3), and this difference was statistically significant (hospital admission: 1.5 vs 4.5 days, p£0.01, ICU admission: 4 vs 8 days, p£0.01). The in-house interval 'from door to ICU' however, did not differ. None of the laboratory results on day of admission to the ICU was associated with survival (Table 1) and also disease severity scores (APACHE and SOFA, Figure 2 C,D) were similar between groups. Taken together, early disease progression (expressed as time interval from symptom onset to hospital admission) was found to be more rapid in patients who did not survive 60 days. Vice versa, a lower prob-
A

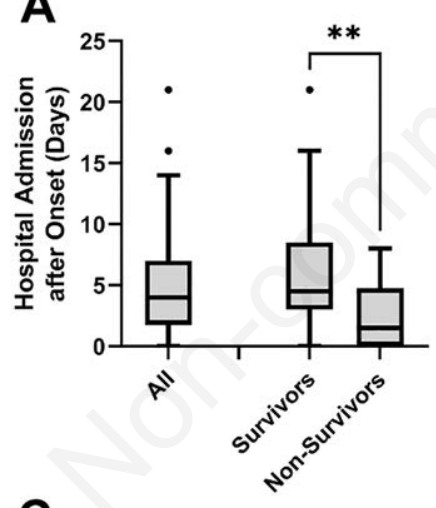

C

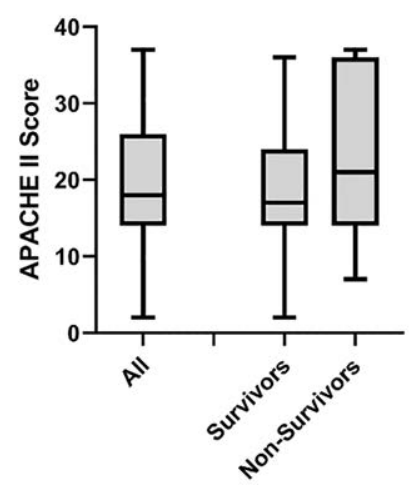

B

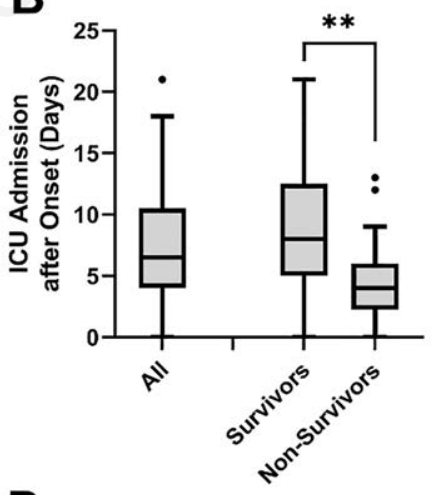

D

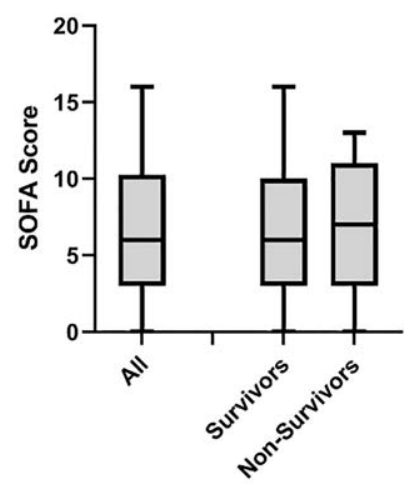

Figure 2. Delay of admission (days after onset of symptoms) and severity of illness on first day in ICU. Patients who died until day 60 were admitted earlier (A) to Hospital ( $\mathrm{p}=\mathbf{0 . 0 0 1 8}$ ), and (B) to ICU ( $\mathrm{p}=\mathbf{0 . 0 0 3 7}$, both Mann Whitney $U$ test) compared to those who survived day 60. Days are counted from ICU admission. (C) APACHE and (D) SOFA scores did not differ significantly (Mann Whitney $\mathrm{U}$ test). Box and whiskers as explained in Figure 1. 
ability of survival was also shown when the total cohort was divided into patients admitted to hospital within 2 days of symptom onset or later (Figure 3).

\section{Discussion}

In our first 50 COVID-19 patients treated on the ICU, we initially focused on the extent of lung damage in comparison with other cohorts. In a retrospective study on 10,021 hospitalized COVID-19 patients in 920 German hospitals, $17 \%$ received mechanical ventilation [20]. Although this proportion of $17 \%$ not necessarily equals the prevalence of ARDS (which was not reported), it is far below ARDS prevalence of about 33\% in 2,486 hospitalized COVID-19 patients in five countries. In this cohort, $63 \%$ of patients needed mechanical ventilation, and ARDS prevalence was $75 \%$ [21]. In our ICU patients, a proportion of $78 \%$ fulfilled the diagnostic criteria for ARDS. Among those who were mechanically ventilated, ARDS prevalence exceeded $90 \%$ and remained on this level in a second assessment the subsequent day. Although being profoundly hypoxaemic, our patients showed comparatively well-preserved lung mechanics, which is rarely seen in patients with 'typical' ARDS. The combination of a large shunt fraction and a rather good lung compliance suggests a novel pathophysiology, thus leading to the hypothesis of gasless tissue being hyperperfused. Accordingly, an increase in oxygenation achieved by PEEP or prone positioning might not primarily result from recruitment but rather from gravity and/or pressure forces [22]. Proning patients with relatively high compliance therefore might not hold much promise. Nevertheless, half of our ventilated patients $(n=22)$ were put in the prone position, and during the first proning period, $p \mathrm{O}_{2}$ rose considerably (Figure 1). Survivors underwent three periods in prone position in median. It has been put forward that autopsy findings of deceased patients with COVID-19 pneumonia matched the original description of ARDS, as diffuse alveolar damage was seen in most cases, notably also in patients who had never been invasively ventilated [23]. Clinically, patients who underwent ECMO therapy presented the 'typical' ARDS findings at this stage of disease: the initially well-preserved lung compliance had finally been lost. This might not only be due to the natural course of the viral pneumonia, but also due to mechanical chal- lenge caused by the great and sustained respiratory efforts of COVID-19 patients before receiving ventilator support. Last but not least, the worsening of lung compliance might also result from positive pressure ventilation itself [24]. In our cohort, NIV or HFNO was rarely used due to the concern of spreading viral aerosols. Furthermore, the distressed patients arriving at our ICUs needed immediate relief from their pronounced shortness of breath - and this included sedation to an extent that precluded an initial non-invasive support. All our patients requiring invasive ventilation were intubated at the latest one day after ICU admission. Since our hospital (like the German health-care system in general) was not overrun by the pandemic at any time during the last months, all patients in need for intensified therapy and mechanical ventilation could be admitted to ICU. In our hospital, all physicians on the wards could call an intensivist 24/7 to discuss the treatment including the possible need to transfer the patient to the ICU. Also, ECMO indication was discussed in an interdisciplinary approach between anaesthesiological and medical ICU specialists. This invasive strategy could be put into action in every case the board had agreed on the indication. Although their number is low, the high in-hospital mortality of our ECMO patients (86\%) compares with the one in the large German retrospective study (71\%) [20]. This exceeds by far the numbers from the ELSO registry, with an in-hospital mortality of 39\% in COVID-19 patients [25]. Although a possible bias cannot be ruled out for these numbers from centres which voluntarily decided to report to the registry, the difference remains remarkable. We did not administer dexamethasone (or any other corticosteroid routinely) to our COVID-19 patients, but, regarding the data available until present, this could be a helpful jigsaw piece in the treatment - although it might be desirable to administer this medication before ICU admission [26]. Remdesivir, as an antiviral agent, does not seem promising for patients needing invasive ventilation [27-29]. As long as a specific antiviral or disease modifying drug is not at hand, the established treatment options against non-COVID-19 ARDS remain our cornerstones taking care of our critical ill COVID-19 patients. About half of our patients could return home until day 60 , and less than half of them reported no limitation on activities on this day $(20 \%$ of the whole cohort). This underlines the medium- and long-term consequences of the disease for patients who needed critical care. The worst outcome ensued for the intubated patients with a parallel need for renal replacement therapy (60-day mortality, 42.1\%).

\section{A Whole Cohort}

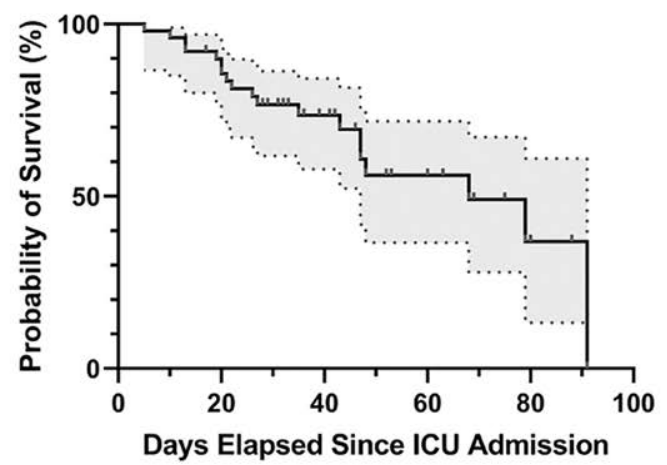

\section{B Early and Late Hospitalization}

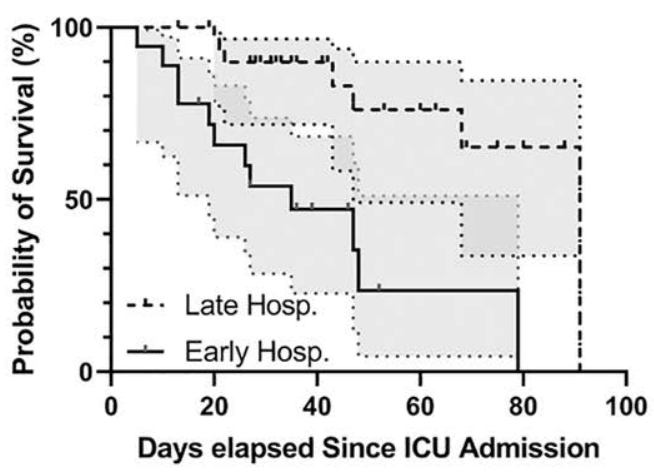

Figure 3. Kaplan Meier estimates of survival (A) in the whole cohort and (B) in the subgroups of patients admitted to hospital within 2 days or later after onset of symptoms. The two survival curves differ significantly (Log-rank test, $\mathrm{p}=\mathbf{0 . 0 0 0 3}$ ). 
Against the backdrop of the German retrospective mega study which offered data on the course of the disease [20], our group of patients showed a comparatively good outcome. Clearly, the time from onset of symptoms to admission to the hospital or to the ICU was significantly lower in the group of non-survivors. It might therefore be concluded that early deterioration of symptoms predicts an unfavourable course of the disease.

Vitamin D values were generally low in our patient cohort. It is still not known whether this is a relevant prognostic factor. It is also unknown whether a substitution (of calcifediol or cholecalciferol) can play a role in treatment of COVID-19 or whether a prophylactic substitution might be warranted. Inverse correlations between vitamin D status and COVID-19 incidence and mortality have been reported in Europe [30,31]. Higher levels of circulating vitamin D have been associated with lower SARS-CoV-2 positivity rates [32]. As vitamin D has pleiotropic actions on the immune system, the supplementation might help to protect against an infection with SARS-CoV-2. In a pilot study, administration of a highdose calcifediol reduced the need for ICU treatment in patients with COVID-19 [33].

The limitations of our study are the retrospective design, the single centre character and the small sample size. The lack of standardised pharmacological treatment also may be an important parameter influencing the outcome. Ordinal scoring of outcome on a 7-point scale might be seen as an improperly simplistic assessment of patients with COVID-19. However, we captured data from all of our first 50 patients, including the outcome score for each of them on day 60 after ICU admission. We feel our data can illustrate the potential harm for COVID-19 patients at any age group, as well as the potential for recovery even for the most severely affected patient group with the need for ventilator and renal replacement therapy.

\section{Conclusions}

Rapid deterioration after onset of symptoms can be seen as an early warning signal for a further unfavourable course of disease and, ultimately, a poor outcome in COVID-19. The consistently low vitamin $\mathrm{D}$ concentration gives reason to take a closer look at the role of vitamin D in COVID-19 patients. As long as there is no specific drug that mitigates the course of COVID-19, lung-protective ventilator therapy including prone positioning remain mainstay of the treatment.

$\begin{array}{ll}\text { Abbreviations } \\ \text { COVID-19, } & \begin{array}{l}\text { coronavirus disease 2019; } \\ \text { intensive care unit; }\end{array} \\ \text { ICU, } & \text { acute respiratory distress syndrome; } \\ \text { ARDS, } & \text { severe acute respiratory syndrome coronavirus 2; } \\ \text { SARS-CoV-2 } & \text { pronic obstructive pulmonary disease; } \\ \text { COPD, } & \text { polymerase chain reaction; } \\ \text { PCR, } & \text { chest computed tomography; } \\ \text { CT, } & \text { sepsis-related organ failure assessment score; } \\ \text { SOFA, } & \text { acute physiology and chronic health evaluation score; } \\ \text { APACHE, } & \text { nontracorporeal membrane oxygenation; } \\ \text { ECMO, } & \text { hinvasive ventilation; } \\ \text { NIV, } & \text { Horovitz index; } \\ \text { HFNO, } & \text { positive endexpiratory pressure. }\end{array}$

\section{References}

1. Richardson S, Hirsch JS, Narasimhan M, Crawford JM, McGinn T, Davidson KW, et al. Presenting characteristics, comorbidities, and outcomes among 5700 patients hospitalized with COVID-19 in the New York City area. JAMA 2020;323:2052-9.

2. Guan WJ, Ni ZY, Hu Y, Liang WH, Ou CQ, He JX, et al. Clinical characteristics of Coronavirus disease 2019 in China. N Engl J Med 2020;382:1708-20.

3. Dreher M, Kersten A, Bickenbach J, Balfanz P, Hartmann B, Cornelissen $\mathrm{C}$, et al. The characteristics of 50 hospitalized COVID-19 patients with and without ARDS. Dtsch Arztebl Int 2020;117:271-8.

4. Yang X, Yu Y, Xu J, Shu H, Xia Ja, Liu H, et al. Clinical course and outcomes of critically ill patients with SARS-CoV-2 pneumonia in Wuhan, China: a single-centered, retrospective, observational study. Lancet Respir Med 2020;8:475-81.

5. Bhatraju PK, Ghassemieh BJ, Nichols M, Kim R, Jerome KR, Nalla AK, et al. Covid-19 in critically ill patients in the Seattle Region — Case series. N Engl J Med 2020;382:2012-22.

6. Arentz M, Yim E, Klaff L, Lokhandwala S, Riedo FX, Chong $\mathrm{M}$, et al. Characteristics and outcomes of 21 critically ill patients with COVID-19 in Washington State. JAMA 2020;323:1612-4.

7. Bellani G, Laffey JG, Pham T, Fan E, Brochard L, Esteban A, et al. Epidemiology, patterns of care, and mortality for patients with acute respiratory distress syndrome in intensive care units in 50 countries. JAMA 2016;315:788-800.

8. Auld SC, Caridi-Scheible M, Blum JM, Robichaux C, Kraft C, Jacob JT, et al. ICU and ventilator mortality among critically ill adults with Coronavirus Disease 2019. Crit Care Med 2020;48:e799-809.

9. Bouch DC, Thompson JP. Severity scoring systems in the critically ill. Continuing Education in Anaesthesia Critical Care \& Pain 2008;8:181-5.

10. Vincent JL, Moreno R. Clinical review: Scoring systems in the critically ill. Crit Care 2010;14:207.

11. Huang C, Wang Y, Li X, Ren L, Zhao J, Hu Y, et al. Clinical features of patients infected with 2019 novel coronavirus in Wuhan, China. Lancet 2020;395:497-506.

12. Wang D, Hu B, Hu C, Zhu F, Liu X, Zhang J, et al. Clinical characteristics of 138 hospitalized patients with 2019 novel Coronavirus-infected pneumonia in Wuhan, China. JAMA 2020;323:1061-9.

13. Spinner CD, Gottlieb RL, Criner GJ, Arribas López JR, Cattelan AM, Soriano Viladomiu A, et al. Effect of remdesivir vs standard care on clinical status at 11 days in patients with moderate COVID-19: A randomized clinical trial. JAMA 2020;324:1048-57.

14. Rothe K, Feihl S, Schneider J, Wallnöfer F, Wurst M, Lukas M, et al. Rates of bacterial co-infections and antimicrobial use in COVID-19 patients: a retrospective cohort study in light of antibiotic stewardship. Eur J Clin Microbiol Infect Dis 2020.

15. Wang Y, Dong C, Hu Y, Li C, Ren Q, Zhang X, et al. Temporal changes of CT findings in 90 patients with COVID-19 pneumonia: A longitudinal study. Radiology 2020;296:e55-64.

16. Ai T, Yang Z, Hou H, Zhan C, Chen C, Lv W, et al. Correlation of chest CT and RT-PCR testing in Coronavirus Disease 2019 (COVID-19) in China: A report of 1014 cases. Radiology 2020;296:e32-40.

17. ARDS Definition Task Force, Ranieri MR, Rubenfeld GD, Thompson BT, Ferguson ND, Caldwell E, et al. Acute respira- 
tory distress syndrome: The Berlin definition. JAMA 2012;307:2526-33.

18. Zádori N, Váncsa S, Farkas N, Hegyi P, Erőss B, Szakó L, et al. The negative impact of comorbidities on the disease course of COVID-19. Intensive Care Med 2020;46:1784-6.

19. Chen L, Yu J, He W, Chen L, Yuan G, Dong F, et al. Risk factors for death in 1859 subjects with COVID-19. Leukemia 2020;34:2173-83.

20. Karagiannidis C, Mostert C, Hentschker C, Voshaar T, Malzahn J, Schillinger G, et al. Case characteristics, resource use, and outcomes of 10021 patients with COVID-19 admitted to 920 German hospitals: an observational study. Lancet Respir Med 2020;8:853-62.

21. Tzotzos SJ, Fischer B, Fischer H, Zeitlinger M. Incidence of ARDS and outcomes in hospitalized patients with COVID-19: a global literature survey. Crit Care 2020;24:516.

22. Gattinoni L, Coppola S, Cressoni M, Busana M, Rossi S, Chiumello D. COVID-19 does not lead to a "typical" acute respiratory distress syndrome. Am J Respir Crit Care Med 2020;201:1299-300.

23. Bos LDJ. COVID-19-related acute respiratory distress syndrome: Not so atypical. Am J Respir Crit Care Med 2020;202:622-4

24. Gattinoni L, Chiumello D, Rossi S. COVID-19 pneumonia: ARDS or not? Crit Care 2020;24:154.

25. Barbaro RP, MacLaren G, Boonstra PS, Iwashyna TJ, Slutsky AS, Fan E, et al. Extracorporeal membrane oxygenation support in COVID-19: an international cohort study of the Extracorporeal Life Support Organization registry. Lancet 2020;396:1071-8.

26. RECOVERY Collaborative Group, Horby P, Lim WS,
Emberson JR, Mafham M, Bell JL. Dexamethasone in hospitalized patients with Covid-19 - Preliminary report. N Engl J Med 2021;384:693-704.

27. Pan H, Peto R, Karim QA, Alejandria M, Henao-Restrepo AM, García CH, et al. Repurposed antiviral drugs for COVID-19interim WHO SOLIDARITY trial results. medRxiv 2020:2020.10.15.20209817.

28. Wang Y, Zhang D, Du G, Du R, Zhao J, Jin Y, et al. Remdesivir in adults with severe COVID-19: a randomised, double-blind, placebo-controlled, multicentre trial. Lance. 2020;395:156978.

29. Beigel JH, Tomashek KM, Dodd LE, Mehta AK, Zingman BS, Kalil AC, et al. Remdesivir for the treatment of Covid-19 Final report. N Engl J Med 2020;383:1813-26.

30. Laird E, Rhodes J, Kenny RA. Vitamin D and inflammation: Potential implications for severity of Covid-19. Ir Med J 2020;113:81.

31. Ilie PC, Stefanescu S, Smith L. The role of vitamin D in the prevention of coronavirus disease 2019 infection and mortality. Aging Clin Exp Res 2020;32:1195-8.

32. Kaufman HW, Niles JK, Kroll MH, Bi C, Holick MF. SARSCoV-2 positivity rates associated with circulating 25-hydroxyvitamin D levels. PLoS One 2020;15:e0239252.

33. Entrenas Castillo M, Entrenas Costa LM, Vaquero Barrios JM, Alcalá Díaz JF, López Miranda J, Bouillon R, et al. Effect of calcifediol treatment and best available therapy versus best available therapy on intensive care unit admission and mortality among patients hospitalized for COVID-19: A pilot randomized clinical study. J Steroid Biochem Mol Biol 2020;203:105751.

Received for publication: 25 December 2020. Accepted for publication: 23 February 2021.

This work is licensed under a Creative Commons Attribution-NonCommercial 4.0 International License (CC BY-NC 4.0).

(C) Copyright: the Author(s), 2021

Licensee PAGEPress, Italy

Multidisciplinary Respiratory Medicine 2021; 16:744

doi:10.4081/mrm.2021.744 